\title{
HISTAMINASE CONTAINING FORMULATION - A NEW APPROACH TO PREVENT FOOD HISTAMINOSIS
}

\section{Mircea Alexandru Mateescu* and Pompilia Ispas-Szabo}

\author{
Research chair on Enteric Dysfunctions "Allerdys" \\ Department of Chemistry and Centre Pharmaqam, Université du Québec à Montreal, \\ CP 8888, Branch A, Montreal (QC), Canada. \\ *mateescu.m-alexandru@uqam.ca
}

\begin{abstract}
Histamine is involved in various regulatory processes and can induce multiple allergic symptoms (hypotension, tachycardia, vascular risks) including anaphylactic shock and possible death. Exogenous histamine, frequently associated to fermented food and beverages, may induce a food histaminosis and trigger pseudo-allergic phenomena for which there is no current treatment available. Histamine may also exert some pro-inflammatory effects, particularly damaging for subjects with Inflammatory Bowel Diseases (IBD) as Crohn's disease (CD) and Ulcerative Colitis (UC) . A novel enzymatic strategy using diamineoxidase (DAO, also called histaminase) to decrease the histamine level in allergic reactions to food and in UC was recently proposed. The therapeutic concept is that histaminase will decrease the level of histamine in the intestinal lumen by oxidative deamination, involving the dissolved oxygen. Since the by-product of the DAO reaction is hydrogen peroxide $\left(\mathrm{H}_{2} \mathrm{O}_{2}\right)$, an association of catalase and vegetal histaminase was proposed. Catalase will decompose $\mathrm{H}_{2} \mathrm{O}_{2}$ generating in situ more oxygen, promoting thus the decomposition of histamine under histaminase action. A recent investigation on intestinal Caco- 2 cells showed a protective role of the bi-enzymatic histaminase-catalase combination. Furthermore, the histological studies have confirmed that suspensions containing histaminase and catalase were able to treat induced colonic inflammation in mice. A formulation with CarboxyMethylStarch (CMS) was also conceived for oral administration and its gastro-protective behavior ensure a colonic delivery of the two enzymes. This enzymatic approach seems a way with no side effects to maintain a good health of the intestinal mucosa and may contribute to improve the treatment IBD and related inflammatory pathologies.
\end{abstract}

Keywords: Medicine, Histamine, Diamine oxidase (DAO, Histaminase), Catalase, Inflammatory Bowel Diseases (IBD), Food histaminosis, Lower intestine and colon delivery, CarboxyMethylStarch

Abbreviations: IBD - Inflammatory Bowel Diseases; DAO - diamineoxidase; CAT- Catalase; CSM - CarboxyMethylStarch; OCT- Organic Cationic Transporters; SGF - Simulated Gastric Fluid; SIF- Simulated Intestinal fluid; UPS - United States Pharmacopeia

\section{Introduction}

The Diamine oxidase (DAO, also known as Histaminase), is a copper-enzyme (EC 1.4.3.22) present in different tissues (kidney, placenta) and in intestinal mucosa where is abundant. It regulates proliferation of cells via degradation (oxidative deamination) of polyamines [1]. DAO also inactivates the endogenous and exogenous excess of food histamine preventing "pseudo-allergic" reactions and food histaminosis.

In healthy body, histamine released from granulocytes and transported in blood stream is usually rapidly inactivated. This mechanism can be disrupted by a low activity of the DAO due to some enteric dysfunctions (i.e. intestinal inflammatory conditions and damaged intestinal wall) . 


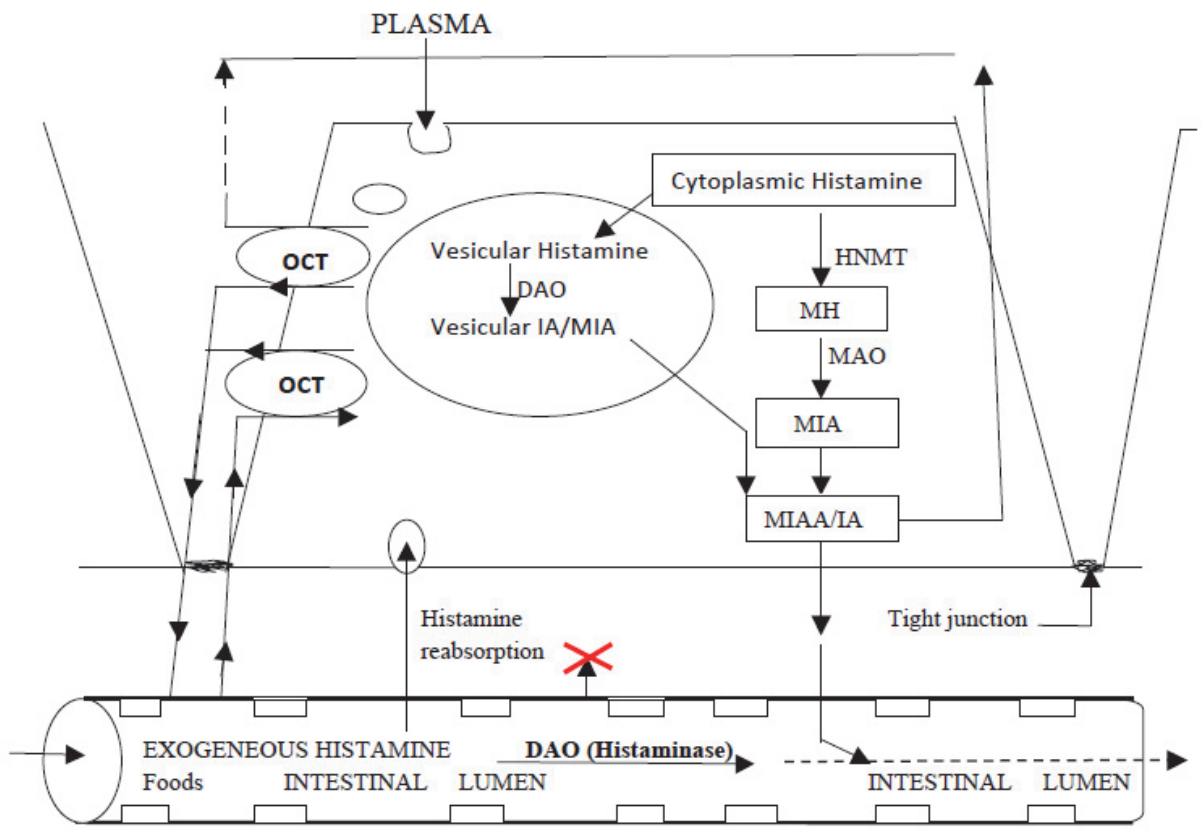

Fig1. 1: Hypothetical presentation of histamine bioelimination by orally administered DAO

Histamine from plasma is uptaken by enterocytes via OCT (Organic Cationic Transporters) and may be catabolized by vesicular DAO (Diamineoxidase) and MAO (Monoamine oxidase) or by HNMT (Histamine methyltransferase) to form IA (imidazole-acetaldehyde), MIA (methyl-imidazoleacetaldehyde), $\mathrm{MH}$ (methyl-histamine) or is eliminated by enterocyte's tight junction in intestinal lumen with possibility of reabsorption (via OCT) by enterocyte cells for further catabolism. Orally administered DAO may decompose Histamine and the re-uptaken is thus prevented (from Mateescu et al., 2017)

Low DAO activity can also be due to a genetic predisposition or inhibition by drugs (i.e. metronidazole [2]. In intestinal bowel diseases (IBD) including Crohn's disease and $\mathrm{UC}$, the level and activity of DAO is reduced, leading to a poor degradation of histamine and to its accumulation which is damaging because histamine is also a pro-inflammatory factor [3]. Current treatment of IBD include anti-inflammatory drugs as non-steroidal antiinflammatory drugs (i.e. 5-aminosalicylic acid mesalazine), corticosteroids, immunesuppressors (infliximab, azathioprine) and antibiotics [4]. These treatments reduce symptoms and may provide remission for various durations but have side effects such as olfaction, drowsiness, nausea, vomiting, dizziness and urinary disorders.

Recently, a novel approach was proposed to treat histamine-related dysfunctions with a vegetal DAO (extract from Lathyrus sativus) in combination with catalase [6]. Catalase is a homo-tetrameric enzyme (EC 1.11.1.6) able to rapidly decompose $\mathrm{H}_{2} \mathrm{O}_{2}$ resulting from the oxidative deamination of histamine by DAO. This approach based on a vegetal DAO associated to Catalase is in development and the bioactive enzymes are formulated for oral administration as monolithic tablets targeted for lower intestine and colon delivery [7].

It was also of interest to investigate to which extent vegetal DAO alone or in combination with catalase, may modulate histamine toxicity in the human intestinal Caco-2 cell line [8]. The effects of histaminerelated molecules, such as histidine (precursor of histamine) and imidazole, were also investigated. 


\section{Materials and Methods}

\subsection{Characterization of DAO and catalase.}

Diamine oxidase was prepared from $500 \mathrm{~g}$ of fresh seedlings of etiolated L. sativus which were homogenized with $1 \mathrm{~L}$ of $\mathrm{NaH}_{2} \mathrm{PO}_{4}(30$ $\mathrm{mM}$ ) and then filtered. The solid residue was washed with the same buffer and the enzyme was finally eluted with $500 \mathrm{~mL}$ of $0.1 \mathrm{M}$ sodium phosphate buffer ( $\mathrm{pH} \mathrm{7)}$ and, then, centrifuged. The supernatant containing the DAO was lyophilized, obtaining thus the DAO vegetal extract powder.

The preparations were characterized for DAO activity as well as for the presence of contaminating-related enzymes that may interfere with the DAO dosage because they can modify the level of $\mathrm{H}_{2} \mathrm{O}_{2}$, namely, catalase (CAT), peroxidase, and superoxide dismutase (SOD) . Diamine oxidase activity was assayed at $25^{\circ} \mathrm{C}$ with $3 \mathrm{mM}$ putrescine in $0.1 \mathrm{M}$ potassium phosphate buffer ( $\mathrm{pH}$ 7.4) by measuring the rate of $\mathrm{H}_{2} \mathrm{O}_{2}$ production forming pink adducts $\left(\varepsilon_{515 \mathrm{~nm}}=2.6 \times 10^{4} \mathrm{M}^{-1}\right.$ $\mathrm{cm}^{-1}$ ) with $2 \mathrm{mM} 4$-aminoantipyrine (AAP) in the presence of horseradish peroxidase (HRP) $(10 \mathrm{U} / \mathrm{mL})$ and of $4 \mathrm{mM} \mathrm{3,5-dichloro-2-}$ hydroxybenzensulfonic acid (DCHBS) . One unit (U) of DAO oxidized $1 \mu \mathrm{mol}$ of putrescine/min. Catalase activity was assayed by measuring the initial decrease in the absorbance of a solution of $15 \mathrm{mM} \mathrm{H}_{2} \mathrm{O}_{2}$ in phosphate buffer (66 mM, pH 7) . One unit of CAT decomposed $1 \mu \mathrm{mol}$ of $\mathrm{H}_{2} \mathrm{O}_{2} / \mathrm{min}$. Peroxidase activity was assayed at $25^{\circ} \mathrm{C}$ in 14 $\mathrm{mM}$ phosphate buffer, $\mathrm{pH} 6$, with $40 \mathrm{mM}$ pyrogallol by measuring the production rate of purpurogallin $\left(\varepsilon 420 \mathrm{~nm}=12 \mathrm{~cm}^{-1}\right)$ in the presence of $8.6 \mathrm{mM} \mathrm{H}_{2} \mathrm{O}_{2}$. One unit of POD produced $1 \mathrm{mg}$ of purpurogallin $/ \mathrm{min}$. Protein content was estimated by the Bio-Rad protein assay reagent using bovine serum albumin as calibration standard.

\subsection{Preparation of bi-enzymatic tablets}

Bi-enzymatic monolithic tablets, based on CMS and CHI (CMS: Chitosan (1: 1, w/w) containing both enzymes (DAO and catalase), were realized with a constant loading of DAO $(20 \%)$ and a variable loading of catalase $(10 \%$ and $20 \%$ ) per tablet. Flat-faced tablets of 300 mg were obtained by direct compression ( 2.5 $\mathrm{T}$ ) of the powder mixtures using $9 \mathrm{~mm}$ cylinder outfits and a Carver press (Wabash, IN, USA).

\subsection{Tablets exposed to simulated gastric fluid and simulate dintestinal fluid}

Tablets of $300 \mathrm{mg}$, based on CarboxyMethylStarch and Chitosan CMS: CHI (1: 1) with $0 \%$ and $50 \%$ enzyme loading or on $100 \%$ enzyme (without excipients), and loaded with $5 \%$ bromocresol green $\mathrm{pH}$ indicator (15 $\mathrm{mg}$ per tablet), were prepared by direct compression of powders.

Each tablet was incubated $60 \mathrm{~min}$ in 50 $\mathrm{mL}$ of SGF containing $0.32 \%$ pepsin (USP, 2017) at $37^{\circ} \mathrm{C}$ and $50 \mathrm{rpm}$ (incubator shaker, series 25D, New Brunswick Scientific Co., USA). The tablet integrity and color change were noticed on the entire and cross-sectioned tablets.

Gastro-protection offered by the polymeric matrix based on CMS: CHI was tested on three series of formulations containing i) DAO only, ii) CAT only or iii) the two enzymes. Tablets were first incubated in Simulated Gastric Fluid (SGF) and then were transferred into $50 \mathrm{~mL}$ Simulated Intestinal Fluid (SIF), $\mathrm{pH}$ 6.8, with $1 \%$ pancreatin (USP, 2017) and incubated at 37 ${ }^{\circ} \mathrm{C}$ and $50 \mathrm{rpm}$.

The total dissolution time of tests in vitro was $24 \mathrm{~h}$, using the same incubator shaker. Samples of $1 \mathrm{~mL}$ were taken from SIF after regular intervals of time, filtered and the DAO and catalase enzymatic activities were determined (as described at 2.1) .

\subsection{Evaluation of DAO enzymatic activity in the presence of catalase}

DAO: CAT powders at different weight ratios $(6: 1,2: 1,1: 1)$ were dissolved in 50 $\mathrm{mL}$ phosphate buffer solution $(50 \mathrm{mM}, \mathrm{pH}$ 7.0), kept under agitation at $4{ }^{\circ} \mathrm{C}$ and filtered prior to determine the DAO enzymatic activity in the presence of catalase. The enzyme activity was determined with the Lglutamate dehydrogenase (GDH) coupled reaction assay (specific for released $\mathrm{NH}_{3}$ assay kit Sigma-Aldrich) The $\mathrm{NH}_{3}$ released from the putrescine substrate (under DAO 
catalysis) reacted with alpha-ketoglutaric acid (KGA) and reduced nicotinamide adenine dinucleotide phosphate (NADPH) in the presence of GDH. The reactional mixture containing $1 \mathrm{~mL}$ of assay kit reagent (KGA and NADPH), $200 \mu \mathrm{L}$ of $30 \mathrm{mM}$ putrescine solution, $10 \mu \mathrm{L}$ of GDH (kit reagent) was incubated for $5 \mathrm{~min}$ at $37^{\circ} \mathrm{C}$ and, finally, 100 $\mu \mathrm{L}$ of DAO samples (containing or not CAT) were added.

The enzymatic reactions were conducted at $37{ }^{\circ} \mathrm{C}$ for $10 \mathrm{~min}$, monitoring the NADPH oxidation to NADP+ by decrease of absorbency at $340 \mathrm{~nm}$. Since CAT also strongly binds NADPH in its active center, its interference was subtracted from each determination of DAO enzymatic activity [6].

\subsection{Cell Culture and viability}

The intestinal Caco-2 cell line obtained following Dr. A Zweibaum procedure [9], was maintained in DMEM containing $25 \mathrm{mM}$ glucose and supplemented with $15 \%$ inactivated $\left(56{ }^{\circ} \mathrm{C}\right.$ for $\left.30 \mathrm{~min}\right)$ fetal bovine serum (FBS), $0.1 \mathrm{mM}$ nonessential amino acids, and $50 \mathrm{U} / \mathrm{mL}-50 \mu \mathrm{g} / \mathrm{mL}$ penicillinstreptomycin. Stock cultures were seeded in $75-\mathrm{cm}^{2}$ culture flasks at $37{ }^{\circ} \mathrm{C}$ in a $5 \% \mathrm{CO}_{2}-$ 95\% humidified air atmosphere and were passaged weekly with trypsinization $(0.05 \%$ trypsin-0.053 mM EDTA). For all the experiments, cells were seeded in 96-well plates $\left(5 \times 10^{3} /\right.$ well $)$. The culture medium was changed every 2 days, and cells were maintained for 7 or 21 days in order to study early confluent but undifferentiated cell cultures and the well-differentiated cells. Cell viability was measured as described before [8] by the MTT colorimetric assay, which measures MTT reduction to a blue formazan product by dehydrogenases of viable cells.

\section{Results}

3.1 Stability of enzyme formulations in simulated gastric fluid and enzyme delivery in simulated intestinal fluid

To the air, the dry tablets based on CMS: CHI (1: 1) unloaded $(0 \%)$ and loaded with $50 \%$ DAO or with CAT presented some blue points at the external surface, corresponding to the $\mathrm{pH}$ indicator (Fig. 3A) . The surface of the entire tablets (with $0 \%$ and $50 \%$ enzyme loading) became blue after $60 \mathrm{~min}$ of acidic incubation (SGF) . The CMS: CHI matrix forms an outer gel barrier, affording thus a certain protection against gastric acidity, despite the fact that the tablets are not coated with gastro-protective materials.

The CMS: CHI polymeric matrix offered a good protection to DAO, with $55.5 \%$ remaining DAO activity found inside the tablet (Fig. 3B) . This matrix could present a potential interest, ensuring a good protection of the DAO even after 120 min incubation in SGF, with maximal release of therapeutic enzyme in SIF at about $8 \mathrm{~h}$ (enough time for tablets to reach the colon). The DAO enzymatic activity consists in oxidation of putrescine in the presence of dissolved $\mathrm{O}_{2}$ (the two substrates of DAO). When CAT is also added, part of $\mathrm{H}_{2} \mathrm{O}_{2}$ produced by DAO will be decomposed generating some supplementary

$\mathrm{O}_{2}$, which is expected to enhance the rate of substrate (histamine, putrescine) oxidation and even to shift theequilibrium in favor of the reaction products (Fig. 2) .

The association of CAT to DAO and their simultaneous release is supposed to be therapeutically beneficial in IBD treatment.

\subsection{Effect of Vegetal DAO on Histamine Cytotoxicity}

The effect of the vegetal DAO on histamine toxicity was studied for 2.75 and 8.5 $\mathrm{mM}$ histamine which led to 25 and $50 \%$ mortality, respectively. DAO at a concentration of $0.77 \mathrm{mg}$ solid $/ \mathrm{mL}$ significantly increased the toxicity of 2.75 or $8.5 \mathrm{mM}$ histamine, lowering cell viability to about $32-38 \%$ in 7-day-old cells (Fig 4) . In all cases, the addition of exogenous CAT at a concentration of $0.77 \mathrm{mg}$ solid $/ \mathrm{mL}$ (7548 $\mathrm{U} / \mathrm{mL}$ ) efficiently protected the cells against DAO-induced histamine toxicity. The protective effect of CAT was very strong in 7day-old cells, where it efficiently decreased not only the DAO-dependent histamine toxicity but also the toxicity of histamine per se. 


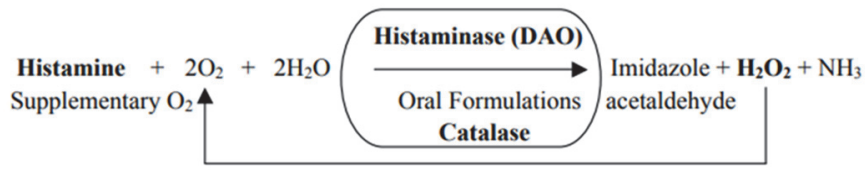

Fig. 2: The enzymatic coupled reactions of DAO and CAT
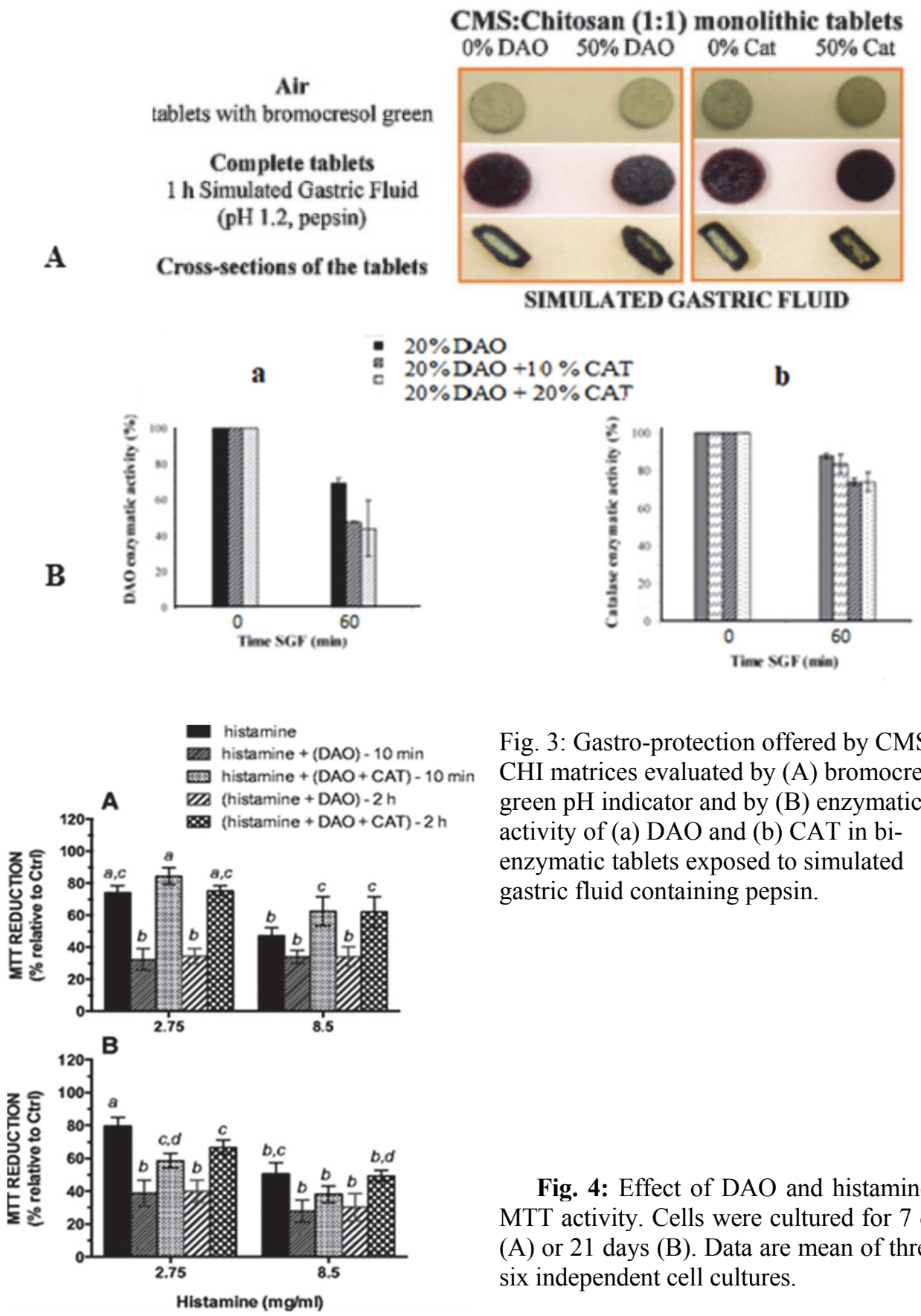

Fig. 3: Gastro-protection offered by CMS: CHI matrices evaluated by (A) bromocresol green $\mathrm{pH}$ indicator and by (B) enzymatic activity of (a) DAO and (b) CAT in bienzymatic tablets exposed to simulated gastric fluid containing pepsin.

Fig. 4: Effect of DAO and histamine on MTT activity. Cells were cultured for 7 days (A) or 21 days (B). Data are mean of three to six independent cell cultures. 


\section{Conclusion}

This study showed the potential of the CMS: CHI formulations to protect DAO and/or CAT against simulated gastric conditions and to control their release in simulated intestinal fluid. The bi-enzymatic DAO: CAT tablets are expected to improve the treatment of inflammatory enteric diseases by reducing local inflammation through accelerated histamine catabolism and by preventing free radical-mediated tissue injury

The present data suggest that the combination of DAO with CAT has great potential to control the biological effect of histamine. Considering the turnover number of the two enzymes, appropriate concentrations are required for a fast removal of histamine without accumulation of $\mathrm{H}_{2} \mathrm{O}_{2}$. The presence of CAT not only promotes the clearance of toxic $\mathrm{H}_{2} \mathrm{O}_{2}$ but, by releasing oxygen, also favors the shift of equilibrium toward histamine decomposition in the DAOcatalyzed reaction.

\section{Acknowledgment}

Financial support from Natural Sciences and Engineering Research Council (NSERC) of Canada (Discovery Grant) and from Fondation Courtois (Canada) is gratefully acknowledged.

\section{References}

B. Mondovì et al., Recent Pat Inflamm. Allergy Drug Discov. 7 (2013) 20.

O. Befani et al., Inflamm. Res. 50S (2001) 136.

L. Maintz, N. Novak, Am. J. Clin Nutr. 85 (2007) 1185.

JK Triantafillidis et al., Drug Des. Devel. Ther. 5 (2011) 18.

MA Mateescu et al., J. Gastroenterol. Res. 1 (2017) 34.

C. Calinescu et al., Int. J. Pharmaceutics 428 (2012) 48.

MA Mateescu et al., US 9878020 (2018) .

C. Jumarie et al. App. Biochem. Biotech 182 (2017) 1171.

I. Chantret et al., Cancer Research 48 (1988) 1936. 